\title{
Relações entre depressão, habilidades sociais, sexo e nível socioeconômico em grandes amostras de adolescentes
}

\author{
Josiane Rosa Campos*, Zilda Aparecida Pereira Del Prette, \& Almir Del Prette \\ Universidade Federal de São Carlos. São Carlos, SP, Brasil.
}

\begin{abstract}
RESUMO - Ainda não se têm claras as relações entre presença de indicadores de depressão, habilidades sociais, sexo e nível socioeconômico em adolescentes. Este estudo comparou uma amostra geral de 642 adolescentes em relação à mesma amostra, porém, sem os participantes com indicadores de depressão (539 adolescentes), buscando verificar o impacto desse controle no que se refere as habilidades sociais, o sexo e o nível socioeconômico. Houve rebaixamento do escore global, das habilidades sociais de empatia e autocontrole, na amostra feminina. Não houve diferença estatisticamente significativa quanto ao nível socioeconômico. Conclui-se sobre a importância de prover os manuais de testes com informações sobre as variáveis controladas e não controladas nas amostras utilizadas para o estabelecimento de padrões normativos.
\end{abstract}

PALAVRAS-CHAVE: adolescentes, depressão, habilidades sociais, nível socioeconômico, grandes amostras

\section{Relating depression, social skills, sex and socioeconomic status in large samples of adolescents}

\begin{abstract}
It is still unclear whether indicators of depression, social skills, sex and socioeconomic status are related in adolescents. This study compared a general sample of 642 adolescents with the same sample, but without the participants with depression indicators (539 adolescents) seeking to verify the impact of this control over social skills, sex and socioeconomic status. The global score of social skills diminished, and also the scores of empathy and self-control in the female sample. There were no statistically significant differences in socioeconomic status. on the results indicate the importance of providing information in tests manuals on the controlled and not controlled variables in the samples used to establish normative standards.

KEYWORDS: adolescents, depression, social skills, socioeconomic level, large samples
\end{abstract}

Atualmente se reconhece uma prevalência de depressão na adolescência, em torno de $20 \%$ (World Health Organization [WHO], 2012). Estudos focalizando variáveis sociodemográficas relacionadas à prevalência de depressão mostram que ela pode ser maior em meninas (Avanci, Assis, \& Oliveira, 2008; Bor, Dean, Najman, \& Hayatbakhsh, 2014) e em adolescentes com menor nível socioeconômico (NSE; Fortenberry, 2003). Resultados semelhantes foram encontrados por Campos (2010) na avaliação de uma amostra de 642 adolescentes, de 12 a 14 anos, nos quais identificou 103 (ou seja, 16\%) com indicadores de depressão, sendo que destes 75 (72,8\%) eram meninas.

A partir dos anos 80 e 90, estudos sobre depressão em adolescentes constataram que estes apresentavam sintomas similares aos dos adultos (Méndez, Olivares, \& Ros, 2005).
Esses sintomas incluem fadiga, tristeza, irritabilidade, dificuldades de apetite (excesso ou falta), problemas com o sono, anedonia bem como alterações somáticas e cognitivas que afetam expressivamente a capacidade de funcionar do indivíduo, com duração de pelo menos duas semanas (American Psychiatric Association [APA], 2014).

A depressão pode ser caracterizada como o aumento de comportamentos com função de fuga-esquiva, como, por exemplo, queixas, reclamações frequentes, choro e a diminuição na frequência de certos comportamentos, como atividades sexuais, hobbies, esportes e engajamento em relacionamentos sociais (Ferster, 1973). Como a adolescência é um período de mudanças importantes nos relacionamentos interpessoais, esse padrão de condutas e sintomas pode afetar drasticamente a qualidade dos

*E-mail: josirosacampos@gmail.com 
relacionamentos dessa população e, por essa via, gerar um ciclo vicioso que compromete ainda mais a sua saúde.

Pesquisando padrões interativos, alguns pesquisadores levantaram a hipótese de que adolescentes deprimidos apresentam déficits de habilidades sociais (Campos, 2010; Fine, Forth, Gilbert, \& Haley, 1991; Reed, 1994, Saldaña, Del Prette, Del Prette, Gasca, \& Treviño, 2006; Young, Mufson, \& Davies, 2006). Adicionalmente, estudos do campo teórico-prático das habilidades sociais também têm produzido conhecimentos relevantes para a compreensão dos padrões interpessoais de diferentes transtornos psicológicos e para o planejamento de programas de prevenção e promoção de saúde do adolescente em geral (Del Prette \& Del Prette, 2009a; Gaspar, 2008; Murta, Del Prette, Nunes, $\&$ Del Prette, 2006; Wagner \& Oliveira, 2015).

De acordo com Del Prette e Del Prette (2006), as habilidades sociais "são diferentes classes de comportamentos sociais do repertório de um indivíduo que contribuem para a competência social, favorecendo um relacionamento saudável e produtivo com as demais pessoas" (p. 31). Nesse sentido, o conceito de habilidades sociais abrange um sentido descritivo dos comportamentos verbais e não verbais, enquanto que o conceito de competência social remete a um sentido avaliativo do desempenho social apresentado por um indivíduo. Para os autores, um desempenho é competente socialmente quando, mantendo coerência entre pensamentos, sentimentos e ações, articula esses componentes de forma bem-sucedida em resposta às demandas pessoais e das diferentes situações sociais, apresentando, então, alta probabilidade de gerar consequências reforçadoras para o indivíduo e para seus interlocutores (Del Prette \& Del Prette, 2006, 2012).

Del Prette e Del Prette (2012) entendem que um bom repertório de habilidades sociais é condição necessária, ainda que não suficiente, para a competência social. No contexto da adolescência, Del Prette e Del Prette (2009b) definiram empiricamente, em estudo psicométrico, seis classes de habilidades sociais importantes para atender às demandas vivenciadas nas relações com pais, , irmãos, amigos, autoridades e desconhecidos: empatia, autocontrole, civilidade, assertividade, abordagem afetiva e desenvolvimento social.

Conforme a literatura da área, as habilidades sociais são aprendidas e aprimoradas ao longo da vida, dependendo das condições ambientais às quais um indivíduo é exposto. $\mathrm{O}$ contexto social e cultural tem influência sobre a aquisição e funcionalidade das habilidades sociais, o que foi detalhado por Del Prette e Del Prette (1999) em termos das dimensões cultural, situacional e pessoal das habilidades sociais, entendidas como níveis que se articulam e se influenciam mutuamente.

As características pessoais que afetam ou são afetadas pelo repertório de habilidades sociais podem incluir variáveis originalmente determinadas pela genética (por exemplo, sexo, inteligência, predisposições para saúde ou doença), mas que podem ser alteradas ou reconstruídas pelo ambiente e a cultura (por exemplo, valores familiares, práticas parentais, disponibilidade de escolarização). Adicionalmente, há variáveis estritamente ambientais, como a condição socioeconômica, que estabelecem condições favoráveis ou desfavoráveis ao estímulo, desempenho e aprendizagem de comportamentos, incluindo as habilidades sociais, ao longo do desenvolvimento (Bandeira, Rocha, Freitas, Del Prette, $\&$ Del Prette, 2006; Del Prette \& Del Prette, 2009b; GerkCarneiro, Dias, Nyaradi, \& Aquino, 2000).

Quanto à relação entre habilidades sociais e NSE, os resultados obtidos por Del Prette e Del Prette (2009b), no estudo de validação de um instrumento de habilidades sociais com a população geral de adolescentes, apontaram correlações positivas, ainda que muito baixas, entre classe social e habilidades sociais da amostra, nos indicadores de frequência e dificuldade. Os autores fazem uma ressalva quanto aos resultados, pois houve uma quantidade baixa de participantes de indivíduos das classes D e E. Todavia, Bandeira et al. (2006) identificaram, na população infantil, que, quanto maior o NSE, maior o repertório de habilidades sociais das crianças. Entretanto, no que se refere a estudos com adolescentes, a revisão de Campos, Del Prette e Del Prette (2014a) revelou que a variável socioeconômica pouco é mensurada junto ao repertório de habilidades sociais, o que sugere a inclusão da mesma nos estudos.

A variável sexo também parece importante de ser incluída nos estudos em habilidades sociais, pois, embora meninos e meninas apresentem características anatômicas que os definam como tais, sabe-se que as diferenças comportamentais entre homens e mulheres claramente começam a ocorrer desde cedo (Afifi, 2007; APA, 2011). As explicações em geral reconhecem o papel da cultura em modelar diferencialmente o repertório social de ambos os sexos (Nolen-Hoeksema, 2012).

Tomando como base a amostragem geral normativa, obtida por conveniência, Del Prette e Del Prette (2009b) encontraram que, considerando indicadores de frequência, as meninas de 12 a 14 anos apresentavam maiores escores de habilidades sociais que os meninos da mesma idade, no escore total e nas subescalas empatia, autocontrole, civilidade e assertividade. Nos indicadores de dificuldade, os resultados também foram mais favoráveis às meninas, que apresentaram menor escore no resultado geral e nas habilidades de empatia, civilidade e assertividade (Del Prette \& Del Prette, 2009b).

Em uma amostra geral de 12 a 17 anos, Comodo (2012) encontrou diferenças significativas no repertório geral e nas habilidades de empatia e civilidade, com desvantagem para os meninos. Resultados semelhantes foram encontrados no estudo português de Monteiro (2011), indicando que as meninas apresentaram vantagem no escore total e nas habilidades de empatia. Porém, há resultados contraditórios 
nos escores de empatia, com alguns estudos indicando maior empatia para meninas (Chow, Holly, \& Duane, 2013; Wolfer, Cortina, \& Jürgen, 2012) e outros, para meninos (McMahon, Wernsman, \& Parnes, 2006).

Quanto à relação entre habilidades sociais e depressão, a revisão efetuada por Campos et al. (2014a) identificou 28 estudos sobre habilidades sociais e depressão em adolescentes. Alguns poucos estudos testaram a hipótese de déficits de habilidades sociais na depressão, usando grupos comparativos de adolescentes. Goldstein, Miklowitz e Mullen (2006), com 18 adolescentes deprimidos (diagnóstico de depressão bipolar) e 18 não deprimidos, ambos os grupos apresentando média de 15 anos, mostraram que os adolescentes sem o diagnóstico apresentavam melhor repertório global e de habilidades de autocontrole e assertividade quando comparados ao grupo com diagnóstico. Importante apontar que o estudo apresentou uma análise geral dos dados e não houve objetivos de analisar o sexo e nem o NSE.

O estudo de Campos, Del Prette e Del Prette (2014b) comparou duas amostras de 103 adolescentes cada, com idades de 12 a 14 anos, uma com e outra sem indicadores de depressão, e realizou análises entre os sexos. Nesse estudo, houve diferenças estatisticamente significativas entre os grupos subclínico (com indicadores de depressão) e não clínico (sem indicadores de depressão) para as meninas no escore geral de habilidades sociais, autocontrole, civilidade e assertividade, com vantagem para o grupo não clínico, no indicador de frequência. Para o indicador de dificuldade, também houve diferenças significativas no escore geral de habilidades sociais, nas classes de empatia, autocontrole e civilidade, com o grupo não clínico a relatar baixo custo de resposta. Para a amostra masculina, houve diferença estatisticamente significativa apenas para a classe desenvoltura social, no indicador de frequência, com vantagem para o grupo não clínico. No indicador de dificuldade, as diferenças entre os grupos ocorreram para as classes assertividade e abordagem afetiva, sendo o grupo não clínico a relatar com baixo custo de resposta.

Os estudos referidos apoiam a hipótese de que depressão e indicadores de depressão podem ser associados a déficits de habilidades sociais em adolescentes, especialmente do sexo feminino. Observa-se também que os estudos citados foram conduzidos com amostras gerais, sem controle de possíveis transtornos psicológicos dos participantes. Pode-se, portanto, supor que incluam um percentual de participantes que apresentam problemas ou transtornos psicológicos, correspondentes à prevalência esperada, conforme estimativas da WHO (2012). No caso específico da depressão não diagnosticada em adolescentes, estudo anterior de Campos (2010) permite supor que aproximadamente $16 \%$ dos participantes apresentariam indicadores de depressão.

Essa relação aponta para a potencial importância do controle de indicadores de depressão, quando se estabelece dados normativos sobre habilidades sociais de adolescentes. Pode-se, portanto, levantar a hipótese de que amostras grandes de adolescentes, obtidas por conveniência, por apresentarem um percentual de participantes com indicadores de depressão, estabelecem escores rebaixados de habilidades sociais, especialmente no caso de mulheres. Contra essa hipótese, pode-se supor que, em amostras grandes, as características de uma proporção pequena de respondentes (no caso, em torno de 16\%) não sejam suficientes para alterar significativamente os valores amostrais normativos ou, ainda, que características diversificadas de subamostras acabem se compensando sem maior impacto sobre tais dados.

No entanto, mesmo se reconhecendo a dificuldade de controlar todas as variáveis em amostras de conveniência, essas hipóteses têm implicações sobre o estabelecimento de normas em estudos avaliativos de habilidades sociais e pode ser tomada como uma questão empírica de interesse para a avaliação de habilidades sociais, a avaliação da depressão e para as implicações desses estudos. Assim, considerando esses aspectos, o presente estudo teve como objetivo comparar uma amostra geral de adolescentes (642 participantes), obtida por conveniência, em relação à mesma amostra, porém, sem participantes com indicadores depressão (539 participantes), visando verificar o impacto desse controle sobre os escores de habilidades sociais e a influência do sexo e do NSE sobre os resultados. O estudo visou também discutir as diferenças de sexo no repertório de habilidades sociais, associadas à depressão, em termos de suas implicações para a prevenção e saúde mental.

\section{MÉTODO}

\section{Questões Éticas}

A pesquisa obteve a aprovação do Comitê de Ética da Universidade Federal de São Carlos (CEP/UFSCar), sob o parecer de ${ }^{\circ} 110 / 2009$ e em cumprimento às normas éticas do Conselho Nacional de Saúde (Resolução 196/96).

\section{Participantes}

Participaram do estudo 539 adolescentes sem indicadores de depressão (grupo não clínico), sendo 250 do sexo feminino e 289 do sexo masculino, e 103 adolescentes com indicadores de depressão (75 adolescentes do sexo 
feminino e 28 do sexo masculino). O grupo geral totalizou 642 participantes, 325 do sexo feminino e 317 do sexo masculino. Os indicadores de depressão foram avaliados por meio do Inventário de Depressão Infantil (Children Depression's Inventory [CDI]). A Tabela 1 explicita as características sociodemográficas da amostra.

Uma análise de variância de um fator (ANOVA one way) mostrou que a amostra geral e a amostra não clínica (tratadas neste estudo como independentes) não apresentaram diferença significativa nessas variáveis. Em outras palavras, as duas amostras são equivalentes quanto $a ̀$ idade $(\mathrm{F}=0,518$, $\mathrm{df}=1179, \mathrm{p}=0,472)$, série $(\mathrm{F}=0,642, \mathrm{df}=1179, \mathrm{p}=0,423)$ e NSE $(F=0,079, d f=1179, p=0,779)$. Essa equivalência se manteve inclusive quando se comparou separadamente a subamostra do sexo masculino (idade: $\mathrm{F}=0,120, \mathrm{df}=605$ $\mathrm{p}=0,730$; série: $\mathrm{F}=0,078, \mathrm{df}=605, \mathrm{p}=0,780$; NSE: $\mathrm{t}=-280$. $\mathrm{df}=605, \mathrm{p}=0,597$ ) e do sexo feminino (idade: $\mathrm{F}=0,417$, $\mathrm{df}=574 \mathrm{p}=0,519$; série: $\mathrm{F}=0,882, \mathrm{df}=574, \mathrm{p}=0,348$; NSE: $\mathrm{F}=0,001, \mathrm{df}=574, \mathrm{p}=0,972$.

\section{Instrumentos}

Inventário de Habilidades Sociais de Adolescentes (IHSADel-Prette; Del Prette \& Del Prette, 2009b). O instrumento apresenta 38 itens que avaliam comportamentos sociais de adolescentes de 12 a 17 anos, em diferentes contextos (família, amigos, colegas, desconhecidos, pessoas de autoridade, parceiros afetivo-sexuais). O respondente deve informar qual a frequência (indicador de frequência) e o quanto é difícil (indicador de dificuldade) apresentar o comportamento social. $\mathrm{O}$ instrumento apresenta seis fatores, nos dois indicadores: F1-Empatia, F2-Autocontrole, F3-Civilidade, F4-Assertividade, F5-Abordagem Afetiva, F6-Desenvoltura Social. Os índices de consistência interna na validação do instrumento (coeficiente alpha de Cronbach), nos indicadores de frequência, foram: Escore total $=0,89 ; \mathrm{F} 1$ $=0,82 \mathrm{~F} 2=0,72 ; \mathrm{F} 3=0,75 ; \mathrm{F} 4=0,67 ; \mathrm{F} 5=0,69 ; \mathrm{F} 6=0,61$.
Os valores obtidos no indicador dificuldade foram: Escore total $=0,90 ; \mathrm{F} 1=0,86 ; \mathrm{F} 2=0,75 ; \mathrm{F} 3=0,83 ; \mathrm{F} 4=0,72 ; \mathrm{F} 5$ $=0,67 ; \mathrm{F} 6=0,51$. O instrumento apresenta propriedades aceitáveis de validade e precisão, sendo recomendado pelo Conselho Federal de Psicologia.

Inventário de Depressão Infantil (Children Depression's Inventory [CDI]). A versão brasileira foi adaptada do original de Kovacs (1992). O CDI rastreia os sintomas depressivos na população infantil e adolescente, com pontos de corte para a faixa etária de 12 a 17 anos. O ponto de corte é de 16 pontos para crianças de 7 a 12 anos e de 19 pontos, para a faixa etária de 13 a 17 anos. Estudo de validação mostrou parâmetros psicrométricos aceitáveis (alfa de Cronbach=0,85) para a população brasileira (Wathier, Dell'Aglio, \& Bandeira, 2008).

Critério Brasil (CCEB, Associação Brasileira de Empresas de Pesquisa, 2003). Avalia os bens de consumo e o nível educacional do chefe de família e classifica a população economicamente, a partir da estimação do poder de compra de consumidores, nas classes em A1, A2, B1, B2, C, D e E.

\section{Procedimento de Coleta de Dados}

Após o consentimento da Secretaria Municipal de Educação para realizar a pesquisa em escolas municipais, os instrumentos foram aplicados pela primeira autora, de forma coletiva, em salas de aulas. A participação dos respondentes se dava mediante a entrega do Termo de Consentimento Livre e Esclarecido (TCLE) assinado pelos pais ou responsáveis.

\section{Análise de Dados}

As respostas dos participantes foram inseridas no software SPSS para Windows versão 19 e foram computados o escore geral e os escores das cinco subclasses do IHSADel-Prette (2009b), para os indicadores de frequência e dificuldade. Também foram inseridos os dados do

Tabela 1

Características sociodemográficas dos grupos não clínico e geral da amostra, considerando o sexo

\begin{tabular}{|c|c|c|c|c|c|}
\hline \multirow[b]{2}{*}{ Grupos e variáveis } & & \multicolumn{2}{|c|}{ Grupo Não Clínico } & \multicolumn{2}{|c|}{ Grupo Geral } \\
\hline & & $\begin{array}{c}\text { Feminino } \\
\mathrm{n}(\%)\end{array}$ & $\begin{array}{c}\text { Masculino } \\
\mathrm{n}(\%)\end{array}$ & $\begin{array}{c}\text { Feminino } \\
\mathrm{n}(\%)\end{array}$ & $\begin{array}{c}\text { Masculino } \\
\mathrm{n}(\%)\end{array}$ \\
\hline \multirow{5}{*}{ Idade } & 12 anos & $45(18,0 \%)$ & $56(19,4 \%)$ & $66(20,3 \%)$ & $65(20,5 \%)$ \\
\hline & 13 anos & $103(41,2 \%)$ & $107(37 \%)$ & $132(40,6 \%)$ & $117(36,9 \%)$ \\
\hline & 14 anos & $102(40,8 \%)$ & $126(43,6 \%)$ & $127(39,1 \%)$ & $135(42,6 \%)$ \\
\hline & Total & 250 & 289 & 325 & 317 \\
\hline & Média (DP) & $13,23(0,73)$ & $13,24(0,75)$ & $13,19(0,74)$ & $13,22(0,76)$ \\
\hline \multirow{3}{*}{ Escolaridade } & $6^{\mathrm{a}}$ série & $44(17,6 \%)$ & $60(20,8 \%)$ & $69(21,2 \%)$ & $69(21,8 \%)$ \\
\hline & $7^{a}$ série & $125(50,0 \%)$ & $148(51,2 \%)$ & $157(48,3 \%)$ & $161(50,8 \%)$ \\
\hline & $8^{a}$ série & $81(32,4 \%)$ & $81(28 \%)$ & $99(30,5 \%)$ & $87(27,4 \%)$ \\
\hline \multirow{6}{*}{ Nível Socioeconômico } & A1 & - & $2(0,7 \%)$ & $3(0,9 \%)$ & $2(0,6 \%)$ \\
\hline & A2 & $24(9,6 \%)$ & $30(10,4 \%)$ & $33(10,2 \%)$ & $31(9,8 \%)$ \\
\hline & B1 & $74(29,6 \%)$ & $74(25,6 \%)$ & $89(27,4 \%)$ & $76(24 \%)$ \\
\hline & B2 & $98(39,2 \%)$ & $96(33,2 \%)$ & $126(38,8 \%)$ & $108(34,1 \%)$ \\
\hline & $\mathrm{C}$ & $53(21,2 \%)$ & $84(29,1 \%)$ & $72(22,2 \%)$ & $97(30,6 \%)$ \\
\hline & D & $1(0,4 \%)$ & $3(0,1 \%)$ & $2(0,6 \%)$ & $3(0,9 \%)$ \\
\hline
\end{tabular}

Nota. $\mathrm{n}$ - tamanho da amostra; (\%) porcentagem da amostra 
Critério Brasil, de acordo com as instruções. Os dados dos respondentes com e sem indicadores de depressão foram codificados como um único grupo (geral) e os dados dos respondentes sem indicadores de depressão foram replicados e codificados como grupo não clínico. Para a análise no presente estudo, os dois grupos foram tratados como independentes. Foram efetuadas comparações entre os dois grupos (teste $t$ de Student, para amostras independentes) para analisar separadamente a amostra masculina e a feminina.

\section{RESULTADOS}

Em relação aos escores de habilidades sociais, obtidos com o IHSA-Del-Prette, foi feita uma análise inicial das duas amostras, sem considerar as diferenças de sexo. A Tabela 2 apresenta os dados descritivos e inferenciais para as análises das habilidades sociais, nos indicadores frequência e dificuldade, considerando conjuntamente os dois sexos, para os Grupos Geral e Não Clínico.

Verificou-se que não houve diferenças entre as duas amostras. Considerando-se conjuntamente os dois sexos, o controle dos respondentes com indicadores de depressão não produziu diferenças estatisticamente significativas em amostragem mais ampla.

A Tabela 3 apresenta somente os dados descritivos e inferenciais dos escores de habilidades sociais, que foram estatisticamente significativos, nos indicadores de frequência e dificuldade, considerando as diferenças de sexo para os Grupos Geral e Não Clínico.

Os resultados mostraram que, no grupo geral, as diferenças de sexo ocorreram principalmente para o indicador de frequência, com as meninas apresentando médias maiores que os meninos no escore total e nas

Tabela 2

Dados descritivos e inferenciais para as análises das habilidades sociais, nos indicadores frequência e dificuldade, considerando conjuntamente os dois sexos, para os Grupos Geral e Não Clínico.

\begin{tabular}{|c|c|c|c|c|c|c|c|c|c|c|c|}
\hline \multirow{2}{*}{ IHSA-Del-Prette } & \multirow{2}{*}{ GRUPO } & \multicolumn{5}{|c|}{ Dificuldade } & \multicolumn{5}{|c|}{ Frequência } \\
\hline & & M & D.P & $\mathrm{t}$ & df & $\mathrm{p}$ & $\mathrm{M}$ & D.P & $\mathrm{t}$ & df & $\mathrm{p}$ \\
\hline \multirow{2}{*}{ ESCORE TOTAL } & $\mathrm{NC}$ & 43,35 & 24,95 & $-1,430$ & 1179 & 0,153 & 93,90 & 26,42 & 1,469 & 1179 & 0,142 \\
\hline & Geral & 45,46 & 25,50 & & & & 91,60 & 27,16 & & & \\
\hline \multirow{2}{*}{ F1-Empatia } & $\mathrm{NC}$ & 9,12 & 8,38 & $-1,269$ & 1179 & 0,205 & 28,51 & 8,53 & 1,194 & 1179 & 0,233 \\
\hline & Geral & 9,76 & 8,66 & & & & 27,91 & 8,72 & & & \\
\hline \multirow{2}{*}{ F2-Autocontrole } & $\mathrm{NC}$ & 11,23 & 6,12 & $-1,231$ & 1179 & 0,219 & 17,33 & 6,86 & 1,508 & 1179 & 0,132 \\
\hline & Geral & 11,68 & 6,41 & & & & 16,71 & 7,09 & & & \\
\hline \multirow{2}{*}{ F3-Civilidade } & $\mathrm{NC}$ & 4,75 & 5,38 & $-1,546$ & 1179 & 0,122 & 17,91 & 5,31 & 1,167 & 1179 & 0,244 \\
\hline & Geral & 5,25 & 5,59 & & & & 17,56 & 5,52 & & & \\
\hline \multirow{2}{*}{ F4-Assertividade } & $\mathrm{NC}$ & 7,11 & 5,76 & $-0,920$ & 1179 & 0,358 & 18,64 & 6,23 & ,955 & 1179 & 0,340 \\
\hline & Geral & 7,42 & 5,85 & & & & 18,21 & 6,35 & & & \\
\hline \multirow{2}{*}{$\begin{array}{c}\text { F5-Abordagem } \\
\text { afetiva }\end{array}$} & $\mathrm{NC}$ & 8,33 & 4,75 & $-0,944$ & 1179 & 0,345 & 11,92 & 5,58 & ,955 & 1179 & 0,340 \\
\hline & Geral & 8,59 & 4,71 & & & & 11,60 & 5,66 & & & \\
\hline \multirow{2}{*}{$\begin{array}{l}\text { F6-Desenvoltura } \\
\text { social }\end{array}$} & $\mathrm{NC}$ & 6,63 & 3,60 & $-0,960$ & 1179 & 0,337 & 10,09 & 4,56 & 1,032 & 1179 & 0,302 \\
\hline & Geral & 6,84 & 3,689 & & & & 9,81 & 4,69 & & & \\
\hline
\end{tabular}

Nota. NC-Não Clínico; M- média; D.P- desvio padrão; t- Teste t de Student; df- grau de liberdade; p-nível de significância estatística.

Tabela 3

Dados descritivos e inferenciais para as análises das habilidades sociais, estatisticamente significativas, nos indicadores de frequência e dificuldade, considerando as diferenças de sexo para os Grupos Geral e Não Clínico

\begin{tabular}{|c|c|c|c|c|c|c|c|c|c|c|c|c|c|}
\hline \multirow{2}{*}{ Escores } & \multicolumn{7}{|c|}{ Grupo Geral } & \multicolumn{6}{|c|}{ Grupo Não Clínico } \\
\hline & Sexo & $\mathrm{n}$ & M & DP & $\mathrm{t}$ & $\mathrm{df}$ & $\mathrm{p}$ & $\mathrm{n}$ & M & $\mathrm{DP}$ & $\mathrm{t}$ & df & $\mathrm{p}$ \\
\hline \multirow{2}{*}{ F-Escore Total } & $\mathrm{F}$ & 325 & 93,74 & 24,14 & 2,02 & 640 & 0,043 & 250 & 98,28 & 21,38 & 3,62 & 537 & 0,000 \\
\hline & M & 317 & 89,40 & 29,81 & 2,02 & & & 289 & 90,10 & 29,62 & 3,70 & & \\
\hline \multirow{2}{*}{ F1-Empatia } & $\mathrm{F}$ & 325 & 29,20 & 7,62 & 3,85 & 640 & 0,000 & 250 & 30,52 & 6,475 & 5,21 & 537 & 0,000 \\
\hline & M & 317 & 26,58 & 9,54 & 3,84 & & & 289 & 26,77 & 9,651 & 5,35 & & \\
\hline \multirow{2}{*}{ F3-Civilidade } & $\mathrm{F}$ & 325 & 18,41 & 4,91 & 3,96 & 640 & 0,000 & 250 & 19,06 & 4,26 & 4,77 & 537 & 0,000 \\
\hline & M & 317 & 16,70 & 5,98 & 3,95 & & & 289 & 16,92 & 5,91 & 4,88 & & \\
\hline \multirow{2}{*}{ F4Assertividade } & $\mathrm{F}$ & 325 & 18,76 & 5,91 & 2,24 & 640 & 0,025 & 250 & 19,68 & 5,35 & 3,66 & 537 & 0,000 \\
\hline & M & 317 & 17,64 & 6,73 & 2,24 & & & 289 & 17,73 & 6,79 & 3,72 & & \\
\hline \multirow{2}{*}{$\begin{array}{c}\text { F5-Abordagem } \\
\text { afetiva }\end{array}$} & $\mathrm{F}$ & 325 & 11,12 & 5,24 & $-2,19$ & 640 & 0,029 & & & & & & \\
\hline & M & 317 & 12,10 & 6,04 & $-2,18$ & & & & & & & & \\
\hline \multirow{2}{*}{ D1-Empatia } & $\mathrm{F}$ & 325 & 9,22 & 8,01 &,- 824 & 640 & 0,039 & 250 & 8,21 & 7,44 & $-2,36$ & 537 & 0,018 \\
\hline & M & 317 & 10,30 & 9,26 &,- 824 & & & 289 & 9,91 & 9,05 & $-2,39$ & & \\
\hline
\end{tabular}

Nota. n-tamanho da amostra; M- média; D.P- desvio padrão; df-grau de liberdade; t- Teste t de Student; df-grau de liberdade; p-nível de significância estatística 
habilidades de empatia, civilidade e assertividade e os meninos apresentando maiores médias nas habilidades de abordagem afetiva. Para o indicador de dificuldade, a diferença de sexo ocorreu somente para a habilidade social de empatia, com maiores médias para os meninos.

Esses dados são similares aos obtidos na amostra normativa do IHSA-Del-Prette (Del Prette \& Del Prette 2009b) em relação ao indicador de frequência, exceto que, na normativa, as meninas apresentaram superioridade também nas habilidades de autocontrole e não se diferenciaram nas de abordagem afetiva e desenvoltura social. Outra diferença em relação aos dados da amostra normativa ocorreu para o indicador de dificuldade, maior para os meninos nas habilidades de empatia, enquanto, na normativa, apresentaram maior dificuldade também no escore geral e nas habilidades de civilidade e assertividade.

Na comparação entre os sexos do grupo não clínico (ou seja, sem os respondentes com indicadores de depressão), as meninas apresentaram maiores médias no escore total, nas habilidades de empatia, civilidade, assertividade. Os meninos, por outro lado, apresentaram maiores médias no indicador de dificuldade de empatia. Na habilidade de abordagem afetiva, não houve diferenciação entre os sexos.
Nas comparações entre os dois grupos, efetuadas separadamente para cada sexo, não se verificaram diferenças no indicador de dificuldade para nenhum dos escores do IHSA-Del-Prette (Del Prette \& Del Prette 2009b). Os dados obtidos na amostra feminina para o indicador de frequência são apresentados na Tabela 4 .

Como se observa na Tabela 4 , no caso das respondentes do sexo feminino, foram verificadas diferenças estatisticamente significativas no indicador de frequência, entre os grupos geral e não clínico, para o escore geral de habilidades sociais e para os escores das classes de empatia e autocontrole, com maiores médias para o grupo não clínico. Observa-se também uma tendência à significância estatística no escore do Fator 4-Assertividade.

A Tabela 5 apresenta os dados descritivos e inferenciais dos respondentes do sexo masculino, no indicador de frequência, para o escore geral e para os escores das seis subescalas.

Observa-se, na Tabela 5, que as médias dos respondentes do sexo masculino dos grupos geral e não clínico não se diferenciaram significativamente, para nenhum dos escores de habilidades sociais.

Tabela 4

Dados descritivos e inferenciais das amostras do Grupo Não Clínico e Grupo Geral do sexo feminino nos escores de habilidades sociais para o indicador de frequência.

\begin{tabular}{|c|c|c|c|c|c|c|}
\hline Escores & Grupos & $\mathrm{n}$ & M & DP & $\mathrm{t}$ & $\mathrm{p}$ \\
\hline \multirow{2}{*}{ Escore Geral } & Não clínico & 250 & 98,28 & 21,38 & $-2,35$ & $0,019 *$ \\
\hline & Geral & 325 & 93,74 & 24,14 & & \\
\hline \multirow{2}{*}{ F1-Empatia } & Não clínico & 250 & 30,52 & 6,47 & $-2,23$ & $0,026^{*}$ \\
\hline & Geral & 325 & 29,2 & 7,62 & & \\
\hline \multirow[b]{2}{*}{ F2-Autocontrole } & Não clínico & 250 & 17,86 & 6,18 & $-2,06$ & $0,039^{*}$ \\
\hline & Geral & 325 & 16,73 & 6,79 & & \\
\hline \multirow{2}{*}{ F3-Civilidade } & Não clínico & 250 & 19,06 & 4,26 & $-1,67$ & 0,094 \\
\hline & Geral & 325 & 18,41 & 4,91 & & \\
\hline \multirow{2}{*}{ F4-Assertividade } & Não clínico & 250 & 19,68 & 5,35 & $-1,92$ & 0,054 \\
\hline & Geral & 325 & 18,76 & 5,91 & & \\
\hline F5-Abordagem & Não clínico & 250 & 11,64 & 5,18 & $-1,18$ & 0,236 \\
\hline Afetiva & Geral & 325 & 8,66 & 5,24 & & \\
\hline F6- Desenvoltura & Não clínico & 250 & 10,33 & 4,31 & $-1,17$ & 0,241 \\
\hline Social & Geral & 325 & 9,8 & 4,51 & & \\
\hline
\end{tabular}

Nota. n-tamanho da amostra; M- média; D.P- desvio padrão; $\mathrm{t}=$ Teste t de Student; $\mathrm{p}$ - nível de significância estatística; asterisco- estatisticamente significativo.

Tabela 5

Dados descritivos e inferenciais das amostras do Grupo Não Clínico e Grupo Geral do sexo masculino nos escores de habilidades sociais para o indicador de frequência.

\begin{tabular}{|c|c|c|c|c|c|c|}
\hline Escores & Grupos & $\mathrm{n}$ & $\mathrm{M}$ & DP & $\mathrm{t}$ & $p$ \\
\hline \multirow{2}{*}{ Escore Geral } & Não clínico & 289 & 90,10 & 29,62 & & \\
\hline & Geral & 317 & 89,40 & 29,81 & $-0,29$ & 0,771 \\
\hline \multirow{2}{*}{ F1-Empatia } & Não clínico & 289 & 26,77 & 9,65 & & \\
\hline & Geral & 317 & 26,58 & 9,54 & $-0,24$ & 0,807 \\
\hline \multirow[b]{2}{*}{ F2-Autocontrole } & Não clínico & 289 & 16,87 & 7,38 & & \\
\hline & Geral & 317 & 16,7 & 7,39 & $-0,96$ & 0,777 \\
\hline \multirow{2}{*}{ F3-Civilidade } & Não clínico & 289 & 16,92 & 5,91 & & \\
\hline & Geral & 317 & 16,7 & 5,98 & $-4,55$ & 0,649 \\
\hline \multirow{2}{*}{ F4-Assertividade } & Não clínico & 289 & 17,73 & 6,79 & & \\
\hline & Geral o & 317 & 17,64 & 6,73 & $-1,69$ & 0,866 \\
\hline F5-Abordagem & Não clínico & 289 & 12,15 & 5,9 & & \\
\hline Afetiva & Geral & 317 & 12,10 & 6,04 & 0,12 & 0,905 \\
\hline F6-Desenvoltura & Não clínico & 289 & 9,87 & 4,75 & & \\
\hline Social & Geral & 317 & 9,72 & 4,88 & $-3,99$ & 0,69 \\
\hline
\end{tabular}

Nota. n-tamanho da amostra; M- média; D.P- desvio padrão; t- Teste t de Student; p-nível de significância estatística. 


\section{DISCUSSÃo}

Os resultados do presente estudo não produziram diferenças estatisticamente significativas entre o grupo geral (sem controle de indicadores de depressão) e o não clínico (excluídos os respondentes com indicadores de depressão), quando se tomou o conjunto dos respondentes de ambos os sexos. No entanto, quando se compararam, em cada grupo, as diferenças de sexo, foram verificadas particularidades do grupo não clínico em relação ao normativo, o que apoia a opção metodológica de comparar duas amostras que diferiam apenas em relação a conter ou não respondentes com indicadores de depressão. Entretanto, novas pesquisas devem ser desenvolvidas para melhor compreender as evidências encontradas neste estudo.

$\mathrm{Na}$ comparação entre os dois grupos deste estudo, tomando-se separadamente a amostra feminina e a amostra masculina, as diferenças significativas do grupo geral e não clínico permitiram verificar o impacto dos indicadores de depressão, maior e mais negativo sobre a amostra feminina. Em outras palavras, apoiam a hipótese deste estudo, de que amostras que contenham respondentes com indicadores de depressão, especialmente do sexo feminino, podem apresentar escores de habilidades sociais mais baixos e, tal como mostra o estudo, em particular, os escores de empatia e autocontrole. O design metodológico aqui utilizado também obteve resultados semelhantes a outros estudos (Campos, 2010; Fine et al., 1991; Goldstein et al., 2006; Reed, 1994; Saldaña et al., 2006; Young et al., 2006).

Em relação ao NSE, os resultados não indicaram impacto para a amostra não clínica nem para a amostra geral, mesmo quando se avaliaram separadamente os respondentes do sexo masculino e feminino. Pode-se, portanto, inferir que, pelo menos nessa faixa etária, as demandas para habilidades sociais e o repertório desses adolescentes de diferentes condições socioeconômicas sejam razoavelmente similares. Esses dados contrariam os obtidos por Bandeira et al. (2006) em estudo com a população infantil, no qual se identificou que quanto maior o NSE, maior o repertório de habilidades sociais.

Pode-se, ainda, supor que a variabilidade amostral em relação ao NSE não foi suficiente na presente amostra para captar esse efeito. Reiteram-se, no entanto, as ressalvas realizadas por Del Prette e Del Prette (2009b) de que nem todas as classes sociais foram contempladas de forma equilibrada na amostra normativa, dificultando uma conclusão mais segura a respeito dessa questão e apontandose para a necessidade de realização de novos estudos com controle sistemático dessa variável.

Em relação às habilidades sociais, as diferenças encontradas no escore geral e nos escores das subclasses de empatia e de autocontrole para o indicador de frequência, mas não para o de dificuldade, parecem sugerir uma maior sensibilidade desse indicador para as respondentes dessa faixa etária, especialmente para aquelas que já apresentam sinais de depressão. Não se trata de afirmar que os respondentes não apresentem dificuldade, em termos de ansiedade ou custo de resposta no desempenho de habilidades sociais (Del Prette \& Del Prette, 2009b), mas que possivelmente esse dado seja mais uniformemente avaliado para adolescentes dessa faixa etária. Trata-se de uma suposição a ser melhor investigada em estudos futuros.

Considerando as habilidades sociais, as diferenças verificadas entre os sexos são compatíveis também com estudos que têm indicado um desenvolvimento mais precoce das meninas em habilidades sociais (Chow et al., 2013; Comodo, 2012; Del Prette \& Del Prette, 2009b; Monteiro, 2011; Wolfer et al., 2012), explicando sua superioridade no início da adolescência em relação aos meninos da mesma idade, com exceção do fator F5-Abordagem afetiva. Esse resultado já havia sido encontrado por Campos et al. (2014b), na comparação entre duas amostras deste estudo, com igual tamanho, comparando o repertório de adolescentes com e sem indicadores de depressão. Os respondentes do sexo masculino apresentaram mais semelhanças e quase nenhuma diferença entre os repertórios, com exceção da classe de desenvoltura social. Uma ressalva importante é que a amostra clínica continha apenas 28 participantes do sexo masculino com indicadores de depressão e a amostra não clínica mais que o dobro de adolescentes masculinos, diferença que pode afetar o poder estatístico da análise efetuada. Esse dado vai ao encontro do pressuposto de que as diferenças entre os sexos refletem o papel da cultura, ao modelar diferentemente comportamentos sociais em homens e mulheres (Afifi, 2007; Nolen-Hoeksema, 2012).

$\mathrm{O}$ presente estudo mostrou diferença, na amostra feminina, especificamente nas habilidades de autocontrole e de empatia. As habilidades de empatia estão intimamente ligadas às gentilezas, ao cuidado com o outro, de identificação de diversos sentimentos das pessoas ao seu redor, ao início de estabelecimento de uma comunicação, de uma relação de amizade (Campos, Del Prette, \& Del Prette, 2014c).

A classe autocontrole, por sua vez, como afirma Skinner (1953/2000), está relacionada ao manejo de conflitos de contingências, que sinalizam, concomitantemente, reforço positivo e negativo, ou seja, diferentes consequências. Em outras palavras, trata-se de escolhas entre consequências de curto e longo prazo que requerem uma resposta autocontrolada, que é parte da cadeia comportamental de resolução de problemas e auxilia na provisão de reforçadores.

Hammen (2009) e Kazdin (1993) apontam que um bom repertório de habilidades sociais é fator que contribui para o adolescente resolver seus problemas diante de situações estressantes, representando, portanto, fator de proteção para transtornos depressivos. Adicionalmente, identificar os contextos em que vivem as adolescentes que apresentam bons indicadores de saúde mental pode ser objeto de investigações futuras. 


\section{CONCLUSÕES}

Os resultados do presente estudo apresentam implicações metodológicas e práticas relevantes para futuras investigações e para programas de saúde destinados a adolescentes. Em ambos os casos, reconhece-se que os dados obtidos podem ser de difícil comparação com os da literatura existente dada a variabilidade metodológica, e mesmo teórica, já referida anteriormente.

Não obstante possíveis limitações, a implicação metodológica imediata dos resultados do presente estudo é apontar para a importância de prover os manuais de testes com informações sobre as variáveis controladas e não controladas nas amostras utilizadas para o estabelecimento de padrões normativos. Os resultados também sinalizam para a importância de amostragens probabilísticas que, de fato, distribuam, de forma aleatória, os indicadores conhecidos em relação à prevalência de indicação de depressão para a população geral. De todo modo, considerase importante reconhecer que a falha em apontar diferenças estatisticamente significativas para a amostra masculina pode ter sido influenciada pelo pequeno número amostral de adolescentes com indicadores de depressão no grupo geral.

As implicações práticas estão relacionadas especialmente aos resultados sobre o repertório de habilidades sociais, uma vez que, no geral, não houve diferenças estatisticamente significativas para os indicadores de dificuldade no desempenho de habilidades sociais e no NSE. A análise apontou que as respondentes do sexo feminino do grupo não clínico apresentam melhores médias de habilidades sociais no escore geral e nos escores de empatia e autocontrole, repertórios que podem ser importantes na promoção de saúde da adolescente. Esses dados podem subsidiar o planejamento de programas cujo objetivo seja prevenir doenças e/ou promover saúde de adolescentes, especialmente em postos de saúde, serviços-escola situados em cursos de graduação em psicologia ou em programas desenvolvidos em escolas, por psicólogos escolares. Essa proposta é coerente com as recomendações dos órgãos mundiais de saúde (Fundo das Nações Unidas para a Infância, 2011; WHO, 2012) em avaliar e promover os recursos pessoais e, em particular, a competência social de adolescentes enquanto alternativa para prevenção de seus transtornos psicológicos e a promoção de sua saúde mental (Senna \& Dessen, 2012; Spence \& Matos, 2000; Steinberg \& Lerner, 2004).

Os dados também sinalizam a importância de intervenções diferenciadas por sexo, dadas às diferenças encontradas. Em termos de pesquisa, pode-se sugerir estudos futuros focalizados na identificação de antecedentes e consequentes de comportamentos sociais, no contexto do adolescente. Essa análise é importante também para uma avaliação mais ampla da competência social dos adolescentes, de ambos os sexos, uma vez que permite identificar consequências de curto e médio prazo que indicam ou sugiram a funcionalidade do desempenho em tarefas de interação social (Del Prette \& Del Prette, 2012).

Enfim, como a amostra foi extraída de uma cidade de aproximadamente 620 mil habitantes do interior de São Paulo, generalizações devem ser feitas com cautela. O Brasil é um país imenso e possui muitas culturas e subculturas que podem apresentar variações nos padrões sociais dos adolescentes, bem como múltiplas diferenças comportamentais entre os sexos.

\section{REFERÊNCIAS}

Afifi, M. (2007). Gender differences in mental health. Singapore Medical Journal, 48(5), 385-391. Retrieved from http://smj. sma.org.sg/4805/4805ra1.pdf

American Psychiatry Association. (2011). The guidelines for psychological practice with lesbian, gay, and bisexual clients. American Psychological Association, 67(1), 10-42 https://doi. org/10.1037/a0024659

American Psychiatry Association. (2014). Diagnostic and statistical manual of mental disorders - DSM-5 (5 ${ }^{\text {th }}$ ed.). Washington: American Psychiatric Association.

Associação Brasileira de Empresas de Pesquisa. (2003). Critério Brasil. Retrieved from http://www.abep.org/criterio-brasil

Avanci, J. Q., Assis, S. G., \& Oliveira, R. V. C (2008). Sintomas depressivos na adolescência: Estudo sobre fatores psicossociais em amostra de escolares de um município do Rio de Janeiro, Brasil. Caderno de Saúde Pública, 24(10), 2334-2346. https:// doi.org/10.1590/S0102-311X2008001000014.

Bandeira, M., Rocha, S. S., Freitas, L. C., Del Prette, Z. A. P., \& Del Prette, A. (2006). Habilidades sociais e variáveis sociodemográficas em estudantes do ensino fundamental. Psicologia em Estudo, 11(3), 541-549. Retrieved from
https://www.researchgate.net/profile/Marina_Bandeira/ publication/222093511_Social_skills_and_sociodemographic variables_in_elementary_school_students_Habilidades_ sociais_e_variaveis_sociodemograficas_em_estudantes_do ensino_fundamental/links/0fcfd50ed68e4d2d9a000000.pdf

Bor, W., Dean, A. J., Najman, J., \& Hayatbakhsh, R. (2014). Are child and adolescent mental health problems increasing in the 21 st century? A systematic review. Australian \& New Zealand Journal of Psychiatry, 8(7) 606-616. https://doi. org/10.1177/0004867414533834.

Campos, J. R. (2010). Habilidades sociais de adolescentes com indicadores de depressão: Considerando fatores de gênero e socioeconômicos (Dissertação de mestrado não publicada). Universidade Federal de São Carlos-UFSCar, São Carlos, SP, Brasil.

Campos, J. R., Del Prette, A., \& Del Prette, Z. A.P. (2014a). Habilidades sociais e depressão na adolescência: Uma revisão da literatura. Acta Comportamentalia, 22(4), 469-482. Retrieved from http://www.redalyc.org/articulo. oa? id=274532646007 
Campos, J. R., Del Prette, A., \& Del Prette, Z. A. P (2014b). Habilidades sociais de adolescentes com e sem indicadores de depressão, considerando análises de sexo (Manuscrito não publicado). Universidade Federal de São Carlos, São Carlos, SP, Brasil.

Campos, J. R., Del Prette, A., \& Del Prette, Z. A. P. (2014c). Depressão na adolescência: Habilidades sociais e variáveis sociodemográficas como fatores de risco/proteção. Estudos e Pesquisas em Psicologia, 14(2), 408-428.

Chow, C. M., Holl, R., \& Duane, B, (2013). The mediating role of interpersonal competence between adolescents' empathy and friendship quality: A dyadic approach. Journal of Adolescence 36(1), 191-200. https://doi.org/10.1016/j. adolescence.2012.10.004 (ME)

Comodo, C. N. (2012). Intergeracionalidade das habilidades sociais entre pais e seus filhos adolescentes (Dissertação de Mestrado). Universidade Federal de São Carlos-UFSCar, São Carlos, SP, Brasil. Retrieved from http://www.ppgpsi.ufscar. $\mathrm{br} /$ defesas/diss-cnc

Del Prette, Z. A. P., \& Del Prette, A. (1999). Psicologia das habilidades sociais: Terapia e educação. Petrópolis: Vozes.

Del Prette, Z. A. P., \& Del Prette, A. (2006). Psicologia das habilidades sociais na infância: Teoria e prática. Petrópolis: Vozes.

Del Prette, A., \& Del Prette, Z. A. P. (2009a). Adolescência e fatores de risco: A importância das habilidades sociais educativas. In V. G. Haase, F. O. Ferreira, \& F. J. Penna (Orgs.), Aspectos biopsicossociais da saúde na infância e adolescência (pp. 503522). Belo Horizonte: Coopmed.

Del Prette, A., \& Del Prette, Z. A. P (2009b). Inventário de habilidades sociais para adolescentes (IHSA-Del Prette): Manual de aplicação, apuração e aplicação. São Paulo: Casa do Psicólogo.

Del Prette, Z. A. P., \& Del Prette, A. (2012). Social skills and behavior analysis: Historical proximity and new issues. Perspectivas em Análise do Comportamento, 1(2), 104-115. Retrieved from http://media.wix.com/ugd/89bfd6_94383ead 2540468482f4156041b37dfb.pdf

Ferster, C. B. (1973). A functional analysis of depression. American Psychologist, 28, 857- 870. doi.org/10.1037/h0035605

Fine, S., Forth, A., Gilbert, M., \& Haley, G. (1991). Group therapy for adolescent depressive disorder: A comparison of social skills and therapeutic support. Journal of the American Academy of Child and Adolescent Psychiatry. 30, 79-85. https://doi.org/10.1097/00004583-199101000-00012

Fortenberry, J. D. (2003). Socioeconomic status, schools, and adolescent depression: Progress in the social epidemiology of adolescent health. The Journal of Pediatrics, 143, 427-429. https://doi.org/10.1067/S0022-3476(03)00467-0

Fundo das Nações Unidas para a Infância. (2011). Saúde mental do adolescente: Um desafio urgente para pesquisas e investimentos. Situação mundial da infância. Retrieved from http://www.unicef.org/brazil/pt/br_sowcr1lweb.pdf

Gaspar, T. (2008). Promoção do bem-estar na adolescência: Impacto do estatuto socioeconômico e do estatuto migrante. Análise Psicológica, 2(XXVI), 256-279. Retrieved from http://www. scielo.mec.pt/pdf/aps/v26n2/v26n2a08.pdf

Gerk-Carneiro, E. G. P., Dias, R. D., Nyaradi, N. O., \& Aquino, S. M. C. (2000). Habilidades sociais e cognitivas em estudantes universitários brasileiros. Revista Galego Portuguesa de Psicologia e Educação, 4(6), 689-697. Retrieved from http:// livrozilla.com/doc/514241/habilidades-sociais-e-cognitivasem-estudantes-universit $\% \mathrm{C} 3 \% \mathrm{~A} 1 \ldots$
Goldstein, T. R., Miklowitz, D. J., \& Mullen, K. (2006). Social skills knowledge and performance among adolescents with bipolar disorder. Bipolar Disorders, 8, 350-361. https://doi. org/10.1111/j.1399-5618.2006.00321.x

Hammen, C. (2009). Adolescent depression stressful interpersonal contexts and risk for recurrence. Current Directions in Psychological Science, 18, 200-204. https://doi.org/10.1111/ j.1467-8721.2009.01636.x

Kazdin, A. E. (1993). Adolescent mental health: Prevention and treatment programs. American Psychologist, 48(2), 127-141. https://doi.org/10.1037/0003-066X.48.2.127

Kovacs, M. (1992). The Children's Depression Inventory, CDI. Toronto: Manual Multi- Health Systems, Inc.

McMahon, S. D., Wernsman, J., \&. Parnes, A. L. (2006). Understanding prosocial behavior: The impact of empathy and gender among african american adolescents, The Journal of Adolescents Health, 39, 135-137. https://doi.org/10.1016/j. jadohealth.2005.10.008

Méndez, F. X., Olivares, J., \& Ros, M. C. (2005). Características clínicas e tratamento da depressão na infância e adolescência. In V. E. Caballo \& M. A. Simón (Orgs.), Manual de psicologia clínica infantil e do adolescente - Transtornos gerais (pp. 139185). São Paulo: Livraria Santos.

Monteiro, M. J. M. (2011). Competências para a vida em adolescentes: Avaliação da qualidade de vida relacionada com a saúde e da competência social (Dissertação de Mestrado). Pós Graduação em Psicologia da Educação, Universidade de Algarve, Gambelas, Portugal.

Murta, S. G., Del Prette, A., Nunes, F.C., \& Del Prette, Z. A. P. (2006). Problemas en la adolescencia: Contribuciones del entrenamiento en habilidades sociales. In J. C. Salamanca (Ed.), Manual de intervención psicológica para adolescentes: Ámbito de la salud y educativo (Unidade 1, Cap. 2). Colombia (Bogotá): PSICOM Editores.

Nolen-Hoeksema, S. (2012). Emotion regulation and psychopathology: The role of gender. Annual Review of Clinical Psychology, 8,161-187. https://doi.org/10.1146/ annurev-clinpsy-032511-143109

Reed, M. K. (1994). Social skills training to reduce depression in adolescents. Adolescence, 29(114), 293-302.

Saldaña, M. R. R., Del Prette, Z. A. P., Del Prette, A., Gasca, A., \& Treviño, L. R. R. L. (2006). Entrenamiento en habilidades sociales para reducir indicadores depresivos en adolescentes mexicanos In L. A. Oblitas (Org.), Manual de Psicología Clínica y de la Salud Hospitalaria (Cap. 48). Colombia (Bogotá): PSICOM Editores.

Senna, S. R. C. M., \& Dessen, M. A. (2012). Contribuições das teorias do desenvolvimento humano para a concepção contemporânea da adolescência. Psicologia: Teoria e Pesquisa, 28(1) 101-108. Retrieved from http://www.scielo.br/pdf/ptp/ v28n1/13.pdf

Skinner, B. F. (2000). Ciência e comportamento humano (J. C. Todorov \& R. Azzi, Trad.). São Paulo: Martins Fontes. (Trabalho original publicado em 1953)

Spence, S., \& Matos, M. (2000). Intervenções preventivas com crianças e adolescentes. In M. Matos, C. Simões, \& S. Carvalhosa (Orgs), Desenvolvimento de competências de vida na prevenção do desajustamento social. Lisboa: IRS/MJ.

Steinberg, L., \& Lerner, R. M. (2004). The scientific study of adolescence: A brief history. The Journal of Early Adolescence, 24, 45-54. https://doi.org/10.1177/0272431603260879

Young, J. F., Mufson, L., \& Davies, M. (2006). Efficacy of interpersonal psychotherapy- adolescent skills training: An 
indicated preventive intervention for depression. Journal of Child Psychology and Psychiatry, 47, 1254-1262. https://doi. org/10.1111/j.1469-7610.2006.01667.x

Wagner, M. F., \& Oliveira, M. S. (2015). Habilidades sociais no abuso e na dependência de maconha. In Z. A. P. Del Prette, A.B. Soares, C. S. Pereira-Guizzo, M. F. Wagner, \& V. B. R. Leme (Orgs), Habilidades Sociais: Diálogos e Intercâmbios Sobre Pesquisa e Prática (pp. 329-347). Editora: SINOPSYS.

Wathier, J. L, Dell'aglio, D. D. E., \& Bandeira, D. R. (2008). Análise fatorial do Inventário de Depressão Infantil (CDI) em amostra de jovens brasileiros. Avaliação Psicológica, 7(1), 75-84. Retrieved from http://pepsic.bvsalud.org/scielo. php?script=sci_arttext\&pid=S1677-04712008000100010\&ln $\mathrm{g}=\mathrm{pt} \& \mathrm{nrm}=\mathrm{iso}>$. ISSN 2175-3431 .

Wolfer, R., Cortina, K. S., \& Jürgen, J. (2012). Embeddedness and empathy: How the social network shapes adolescents' social understanding. Journal of Adolescence, 35(5), 1295-1305. https://doi.org/10.1016/j.adolescence.2012.04.015.
World Health Organization. (2012). Adolescent mental health: Mapping actions of nongovernmental organizations and other international development organizations. Geneva: Author. Retrieved from http://apps.who.int/iris/bitstre am/10665/44875/1/9789241503648_eng.pdf
Recebido: 05/11/2014

Revisado: 14/03/2016

Aceito: 08/05/2016 